
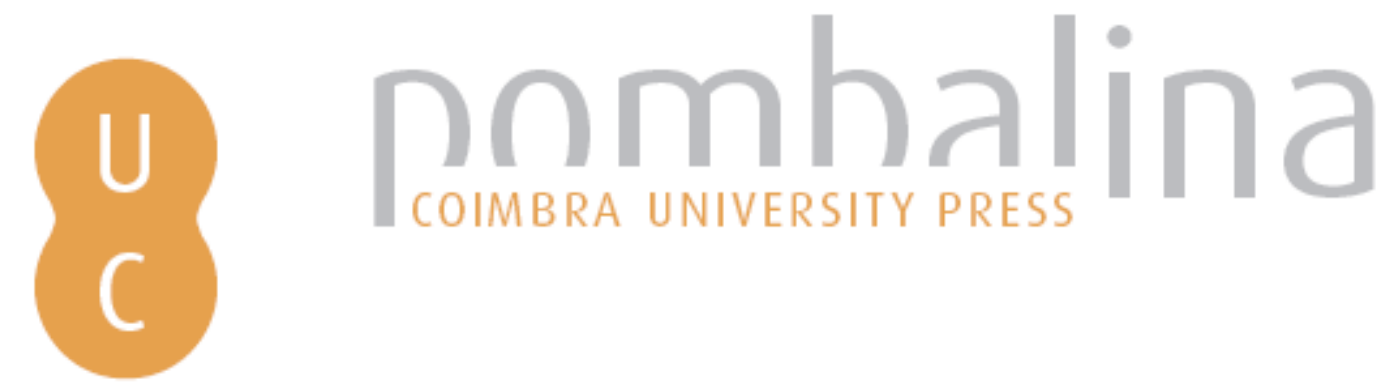

\title{
La ciencia de la información comprendida como un sistema de información y documentación: la aplicación del paradigma emergente
}

Autor(es): $\quad$ Flechas, Eduardo Mancipe; Lukomski, Andrzej

Publicado por: Imprensa da Universidade de Coimbra

URL

persistente: URI:http://hdl.handle.net/10316.2/31880

DOI: $\quad$ DOI:http://dx.doi.org/10.14195/978-989-26-0319-3_16

Accessed : $\quad$ 26-Apr-2023 14:18:32

A navegação consulta e descarregamento dos títulos inseridos nas Bibliotecas Digitais UC Digitalis, UC Pombalina e UC Impactum, pressupõem a aceitação plena e sem reservas dos Termos e Condições de Uso destas Bibliotecas Digitais, disponíveis em https://digitalis.uc.pt/pt-pt/termos.

Conforme exposto nos referidos Termos e Condições de Uso, o descarregamento de títulos de acesso restrito requer uma licença válida de autorização devendo o utilizador aceder ao(s) documento(s) a partir de um endereço de IP da instituição detentora da supramencionada licença.

Ao utilizador é apenas permitido o descarregamento para uso pessoal, pelo que o emprego do(s) título(s) descarregado(s) para outro fim, designadamente comercial, carece de autorização do respetivo autor ou editor da obra.

Na medida em que todas as obras da UC Digitalis se encontram protegidas pelo Código do Direito de Autor e Direitos Conexos e demais legislação aplicável, toda a cópia, parcial ou total, deste documento, nos casos em que é legalmente admitida, deverá conter ou fazer-se acompanhar por este aviso.

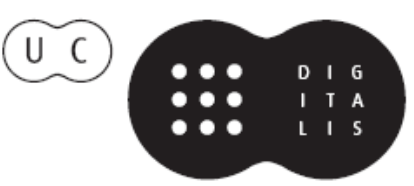


Maria Manuel Borges

Elias Sanz Casado

Coordenação

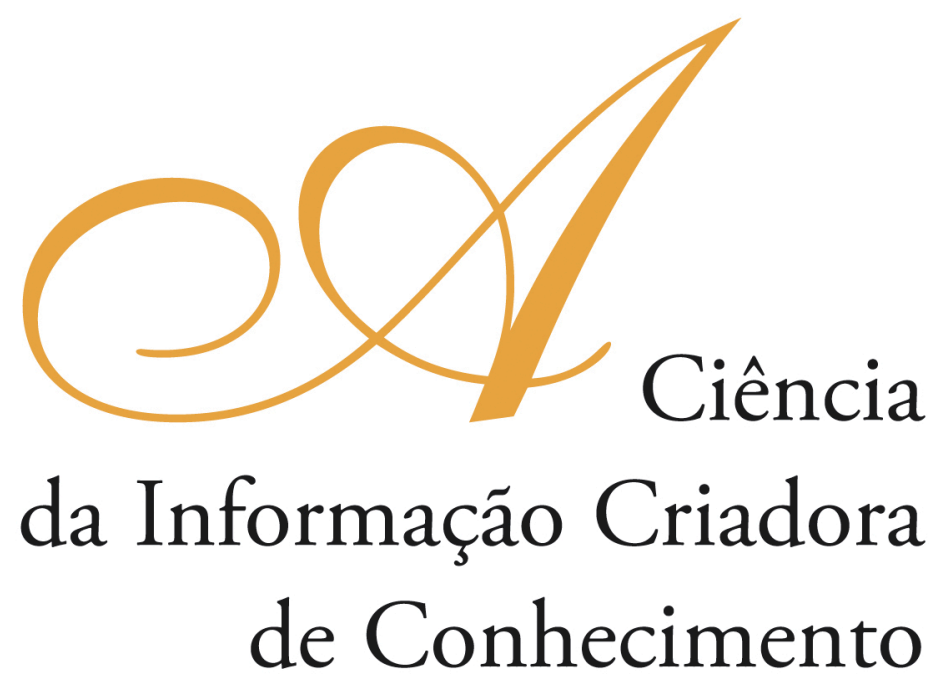

Vol. I

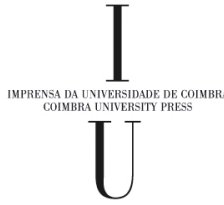

- COIMBRA 2009 


\title{
La Ciencia de la Información comprendida como un Sistema de Información y Documentación: LA APLICACIÓN DEL PARADIgMa EMERgente
}

\author{
Eduardo Mancipe Flechas \\ Universidad de La Salle (Colômbia) \\ Andrzej Lukomski \\ Universidad de La Salle (Colômbia)
}

\section{Resumen}

El presente trabajo pretende mostrar cómo el paradigma emergente -una nueva teoría de la racionalidad propuesta por Beynam y retomada por Miguel Martínez- brinda elementos importantes para la fundamentación epistémica de la Ciencia de la Información comprendida como un Sistema de Información y Documentación -SID-. Para tal fin en primer lugar se realizará una aproximación conceptual de lo que se entiende por SID retomando la propuesta de Miguel Angel Rendón; luego se analizará el alcance de los paradigmas más relevantes adoptados por esta disciplina siguiendo la concepción de paradigma emergente; para finalmente mostrar cómo esta nueva propuesta que se basa en la tendencia al orden en los sistemas abiertos, en una ontología sistémica y en una complementariedad de los enfoques, ofrece una mayor comprensión de los aspectos epistémicos de la Ciencia de la Información.

\begin{abstract}
This paper aims to show how the emerging paradigm, -a new theory of rationality proposed by Beynam and returned by Miguel Martinez- provides important elements in the epistemic foundation of Information Science, including an Information and Documentation System-SID. To this end first will be a conceptual approximation of what is meant by taking the proposed SID Miguel Angel Rendon, then examine the extent of the most important paradigms adopted by the discipline to follow the design of emerging paradigm, to show how this new proposal is based on the tendency to order in open systems, in a systemic ontology and a complementarity of approaches, provides greater understanding of the epistemic aspects of Information Science.
\end{abstract}

\section{Introducción}

El análisis crítico de la mentalidad positivista ha suscitado circunstancias favorables que contribuyan a repensar de modo objetivo los problemas que atañen al valor y alcance del conocimiento científico; los aportes que pretenden situar la actividad científica en un contexto real se han multiplicado no solamente desde disciplinas como la lógica y la filosofía de la ciencia, sino además desde otras disciplinas enmarcadas dentro del área de las ciencias sociales y humanas, como la historia, la psicología, la sociología, la ciencia de la información, entre otras. 
Esta emergencia de un diálogo interdisciplinar en torno a la estructura epistemológica que sustenta el carácter científico de las Ciencias Sociales y Humanas y en particular de la Ciencia de la información, demanda determinar la naturaleza de esta ciencia, proceso cuyo carácter es fundamentalmente descriptivo, lo cual supone el desarrollo de esquemas, interpretaciones y valoraciones que no resultan de una simple recolección de datos, sino del análisis objetivo de los procedimientos empleados por esta disciplina, adoptando una perspectiva meta-científica que se sitúe más allá de la misma, lo cual implica ejercitar una reflexión estrictamente filosófica, que ha de articular los hallazgos proporcionados por los estudios históricos, sociológicos, psicológicos como base para un estudio filosófico de la ciencia.

Lo anterior impulsa a repensar desde un nuevo tipo de racionalidad, la sugestiva propuesta epistemológica de Miguel Angel Rendón en la que propone como objeto de estudio de la Ciencia Bibliotecológica y de la Información, al Sistema de Información y Documentación (SID) conformado por cuatro elementos: a) la información, b) el documento, c) los usuarios y sus necesidades de información, y d) las Instituciones Informativas Documentales, así como el complejo conjunto de sus interrelaciones.

\section{Análisis crítico de la investigación científica basada en el paradigma moderno.}

Frente el paradigma que ofrece la ciencia moderna el investigador puede tomar dos actitudes: Una, aceptar un paradigma sin sentido crítico, a menudo hasta sin ser consciente de ello. La otra posibilidad es la de una actitud crítica, que puede decidir rechazarla. Pero según Popper tenemos que conocer y comprender un paradigma antes de poder decir: "Rechazamos este paradigma por motivos racionales" (1994: 159).

Es posible identificar muchos puntos que ponen en duda el paradigma moderno. Parte de este paradigma es por ejemplo la idea del determinismo. Las personas que no están de acuerdo con el determinismo habitualmente son miradas con sospecha por los modernistas de corte positivista, quienes temen que si aceptamos el indeterminismo, podemos vernos obligados a aceptar muchas de las teorías metafísicas "depuradas" durante la época de ilustración por el pensamiento científico, sin ningún sentido crítico. (Popper, 1994: 159)

La investigación científica, dentro del paradigma moderno, quedó demarcada por las propuestas del positivismo. El positivismo, se basa en el análisis estadístico de los datos recogidos por medio de estudios y experimentos descriptivos y comparativos. Asume que sólo el conocimiento obtenido a través de medidas y de identificaciones objetivas puede presumir de poseer la verdad. (Ruiz, 2007: 12)

El término paradigma desborda los límites fijados por Kuhn (1992), no se limita a cada una de las disciplinas científicas, sino que incluye la totalidad de la ciencia y su racionalidad, razón por la cual Martínez llega a considerar que no son los paradigmas de las ciencias los que están en crisis, sino más bien el paradigma de la ciencia en cuanto modo de conocer. No solamente estamos ante una crisis de los fundamentos del conocimiento científico, sino también del filosófico y en, general ante una crisis de los fundamentos del pensamiento (1997:17). 
La aspiración propia de un investigador - parafraseando a Popper- es reunir todos los aspectos verdaderos del mundo (y no solamente los científicos) en una imagen unificadora que le ilumina a él y a los demás, que pueda convertirse algún día en parte de una imagen aún más amplia, una imagen mejor, más verdadera (Popper,1985: 222).

Un análisis crítico del inventario de las quince características de la ciencia fáctica propuestas por Bunge, nos permitirá determinar el alcance de las mismas desde el horizonte propuesto por un nuevo tipo de racionalidad propuesto por el paradigma emergente.

1. "El conocimiento científico es fáctico: parte de los hechos, los respeta hasta cierto punto y siempre vuelve a ellos. La ciencia intenta describir los hechos tales como son independientemente de su valor emocional o comercial: la ciencia no poetiza los hechos ni los vende, si bien sus hazañas son una fuente de poesía y de negocios. En todos los campos, la ciencia comienza estableciendo los hechos; esto requiere curiosidad impersonal, desconfianza por la opinión prevaleciente, y sensibilidad a la novedad" (Bunge, 2004, 21)

Aquí observamos la imposibilidad de establecer el estatuto científico para todo conocimiento de carácter social, el cual no parte de los hechos sino de la comprensión de los mismos. En el conocimiento social no podemos tener ningún tipo de hechos "puros" analizados de manera impersonal, no tenemos una antropología, sino las antropologías, no tenemos una sociología, sino las sociologías, todo esto muestra que siempre existen elementos personales en el análisis de los hechos. El principio que nos presenta Bunge es un ejemplo de prejuicio de presupuesto que falsifica el verdadero desarrollo del conocimiento científico. Este presupuesto fue establecido desde el paradigma moderno de la ciencia y limita el conocimiento científico a los métodos analíticos, sin tomar en cuenta, el componente hermenéutico de cada pensamiento científico. En la Ciencia de la información, este componente hermenéutico es fundamental y sin él es imposible cualquier tipo de conocimiento. La información siempre está acompañada por la comprensión y los métodos de análisis de la información y exige métodos que se fundamentan en la hermenéutica. Esta falacia positivista ha desviado por mucho tiempo la investigación en el campo disciplinar a cuestiones puramente técnicas y operacionales, frenando la posibilidad de pensar sobre el estatus epistemológico de estas disciplinas porque todo se reducía a técnicas de recuperación de información sin tener en cuenta, el momento de generación del conocimiento en dichos campos de investigación.

2. "El conocimiento científico trasciende los hechos: descarta hechos, produce nuevos hechos y los explica". (Bunge, 2004, 22)

Aquí es evidente que nos falta una actividad muy importante que es comprender los hechos. Esta característica reduce la ciencia únicamente al nivel de explicación y se mueve alrededor del mito de la pura objetividad. Este mito es otro obstáculo que impide establecer el estatus epistemológico de la Ciencia de la Información, dentro del pensamiento moderno.

3. "La ciencia es analítica, la investigación científica aborda problemas circunscritos uno a uno, y trata de descomponerlo todo en elementos. (...) Los problemas de las ciencias son parciales y así son también por consiguiente sus soluciones”. (Bunge, 2004, 24)

Desde la perspectiva del pensamiento complejo este postulado no solamente es falso, sino además perjudicial para el desarrollo de la ciencia. Morin considera este 
supuesto como un supuesto mutilante frente al desarrollo de la ciencia, detrás de esta forma de pensar se esconde todo reduccionismo. Aquí está la razón de atomización de la ciencia y la construcción de la "torre de Babel" del conocimiento científico, tenemos especializaciones tan grandes, lenguajes tan específicos que la comunidad científica pierde la comunicación y el desarrollo del pensamiento científico cae en los laberintos de la especificidad.

La ciencia de la información es emergente es decir surge desde el encuentro de varios tipos de investigación específica, pero por su naturaleza trascienden el ambiente de especificidad y conduce a campos multi-inter-transdiciplinarios, que tienen una descripción interesante en el pensamiento complejo.

4. "La investigación científica es especializada: Una consecuencia del enfoque analítico de los problemas es la especialización. No obstante la unidad del método científico, su aplicación depende en gran medida del asunto; esto explica la multiplicidad de técnicas y la relativa independencia de los diversos sectores de la ciencia”. (Bunge, 2004, 25)

Desde el pensamiento complejo no compartimos la exigencia según la cual, la investigación científica tiene que ser especializada, pero encontramos en el pensamiento moderno algo interesante que es necesario destacar, y es el reconocimiento de la multiplicidad de las técnicas que se concentran en torno a un objeto de investigación, dicha multiplicidad de técnicas debe conducir a la multiplicidad de métodos de la investigación que dinamizan los procesos científicos de la disciplina.

5. "El conocimiento científico es claro y preciso: sus problemas son distintos, sus resultados son claros”. (Bunge, 2004, 26)

En nuestra opinión este postulado no es ningún principio del conocimiento, es un postulado de carácter estético que se impone al pensamiento científico, pero no es substancial para generar los conocimientos.

6. "El conocimiento científico es comunicable: no es infalible sino expresable, no es privado sino público. El lenguaje científico comunica información a quien quiera haya sido adiestrado para entenderlo. (...) La comunicabilidad es posible gracias a la precisión y es a su vez una condición necesaria para la verificación de los datos empíricos y de las hipótesis científicas." (Bunge, 2004, 29).

Estamos de acuerdo con el postulado de la comunicabilidad, no la unificamos con el postulado de la precisión, mas bien consideramos que esa comunicabilidad debe estar enmarcada en el horizonte de sentido, que implica una comprensión más amplia que la precisión.

7. “El conocimiento científico es verificable: Debe aprobar el examen de la experiencia. A fin de explicar un conjunto de fenómenos el científico inventa conjeturas fundadas de alguna manera en el saber adquirido. Sus suposiciones pueden ser cautas o audaces, simples o complejas". (Bunge, 2004, 30)

A este criterio se contrapone la propuesta de Popper, quien considera que el conocimiento científico debe ser falsificable no verificable. Desde esa teoría, la Ciencia de la información no surge de los hechos de la información, ni de las técnicas de la información, sino de las teorías que comprenden la información y desde esas teorías se ven los hechos y establecen las técnicas. El postulado de falsación de Popper permite la libre invención de las teorías que por la naturaleza son vinculadas al conocimiento científico, porque a partir de ellas se genera el conocimiento y la investigación supera el problema de las técnicas de recolección de la información. 
8. "La investigación científica es metódica”. (Bunge, 2004, 31)

Este postulado lo compartimos pero el hecho de que la investigación científica sea metódica no puede reclamar un único método de investigación.

9. "El conocimiento científico es sistemático”. (Bunge, 2004, 33)

Para nosotros es sistemático y sistémico, ampliamos este horizonte.

10. "El conocimiento científico es general: ubica los hechos singulares en pautas generales, los enunciados particulares en esquemas amplios”. (Bunge, 2004, 35).

Este postulado no es solamente el postulado del paradigma moderno de la ciencia, es un postulado de la ciencia que trasciende todos los paradigmas, este era el modo de hacer ciencia en la antigüedad y para nosotros es obvio que las teorías e hipótesis que se logran desde el nuevo paradigma de la ciencia tienen que expresarse en esquemas más amplios que los de los enunciados particulares.

11. "El conocimiento científico es legal: busca leyes de la naturaleza y de la cultura y las aplica. (...) Hay leyes de hechos y leyes mediante las cuales se pueden explicar otras leyes. El principio de Arquímedes pertenece a la primera clase; pero a su vez puede deducirse de los principios generales de la mecánica”. (Bunge, 2004, 36)

Este principio se conserva en el paradigma emergente de la ciencia, no objetamos que hay leyes de hechos y leyes mediante las cuales se pueden explicar otras leyes. Nuestra propuesta es ampliar el campo de investigación para establecer leyes de hechos y no cerrarnos a una visión reduccionista del hecho y leyes de hechos, hay leyes no solamente sobre los hechos sino sobre los fenómenos y la comprensión de los fenómenos. El paradigma emergente no retira las exigencias frente al conocimiento científico, su intención es hacer posible la generación de nuevos conocimientos liberándonos de los mitos, prejuicios y presupuestos que hacen imposible desarrollar tal conocimiento, lo que demanda superar las exigencias que no tienen carácter científico, como la limitación exclusiva a los hechos, dejando de lado los fenómenos y el papel determinante del componente interpretativo.

12. "La ciencia es explicativa: Intenta explicar los hechos en términos de leyes y las leyes en términos de principios. Los científicos no se conforman con descripciones detalladas". (Bunge, 2004, 38)

Esta exigencia restringe demasiado el campo de la investigación científica, la naturaleza de toda investigación de carácter social, requiere descripciones detalladas y análisis de fenómenos, esta es la naturaleza del objeto de investigación, la cual no tiene cabida en la investigación positivista, imponiendo un obstáculo fuerte para el desarrollo de la ciencia. Este postulado de Bunge es un ejemplo de cómo se quiere limitar el desarrollo del conocimiento científico a una concepción muy reduccionista que se impone para validar el conocimiento.

13. "El conocimiento científico es predictivo: Trasciende la masa de los hechos de experiencia, imaginando cómo puede haber sido el pasado y como podrá ser el futuro. La predicción, es, en primer lugar, una manera eficaz de poner a prueba las hipótesis; pero también es la clave de control o aún de la modificación del curso de los acontecimientos". (Bunge, 2004, 39)

Este postulado para nosotros se ubica más en la esfera de los deseos que salen desde la intención de manipular los hechos, pero en nuestra opinión la generación del conocimiento no necesariamente tiene que tener carácter predictivo, el carácter interpretativo, comprensivo es tan igualmente válido como el predictivo. Esto significa 
que la Ciencia de la información no necesita predecir, su valor radica en la profundización de la comprensión e interpretación de la información, conocer las condiciones de la información es no menos válido que conocer la misma información.

14. "La ciencia es abierta, no reconoce barreras a priori que limiten el conocimiento" (Bunge, 2004, 41)

Esto es para nosotros clave, en el paradigma moderno, vemos barreras a priori que limitan el conocimiento. ¿Por qué la Ciencia de la Información no tiene estatus científico en el positivismo? Porque existen barreras a priori desde el paradigma moderno que hacen imposible establecer este horizonte de sentido para la investigación científica. Este campo genera nuevos conocimientos, pero las barreras a priori establecidas por la modernidad hacen que este conocimiento sea reducido a técnicas y tecnologías operacionales.

15. "La ciencia es útil: porque busca la verdad, la ciencia es eficaz en la provisión de herramientas para el bien y el mal” (Bunge, 2004, 49).

Esta es también una característica de la ciencia, pero no de carácter substancial, sino accidental y con clara inclinación frente a las tecnologías y campos de aplicación de conocimiento científico en producción de los bienes y herramientas, lo que es valioso, pero no necesario en el reconocimiento del estatus científico de las disciplinas.

\section{El paradigma emergente}

"El modelo de ciencia que se originó después del Renacimiento sirvió de base para el avance científico y tecnológico de los siglos posteriores. Sin embargo, la explosión de los conocimientos, de las disciplinas, de las especialidades y de los enfoques que se han dado en el siglo XX y la reflexión epistemológica, encuentran ese modelo tradicional de ciencia no sólo insuficiente, sino, sobre todo, inhibidor de lo que podría ser un verdadero progreso, tanto particular como integrado, en las diversas áreas del saber" (Martínez, 2006, p. 726).

Actualmente, según muchos autores, estamos en el umbral de un nuevo paradigma de la racionalidad. Nace así, una nueva concepción de la "objetividad científica", basada también en una diferente teoría de la racionalidad, que pone de relieve el carácter complementario, interdisciplinar y no contradictorio de las ciencias experimentales, que crean y manipulan sus objetos; y las ciencias humanas, que tienen como problema la descripción del sentido que descubren en las realidades. Es lo que algunos autores, por ejemplo Snow (1977) y Prigogine (1986) han venido llamando la Tercera cultura es decir: “...un medio donde pueda realizarse el diálogo indispensable entre los progresos realizados en el modelo matemático y la experiencia conceptual y práctica de economistas, biólogos, sociólogos, demógrafos, médicos que tratan de describir la sociedad humana en su complejidad (Prigogine, 1986: 39)"

Esta teoría de la racionalidad o esquema de comprensión e inteligibilidad de la realidad, en general, y del comportamiento humano en particular, constituye un paradigma emergente, es decir, un paradigma que brota de la dinámica y dialéctica histórica de la vida humana y se impone cada vez con más fuerza y poder convincente, a nuestra mente inquisitiva.

La ontología sistemática y su consiguiente metodología interdisciplinaria cambian radicalmente, la conceptualización de toda identidad. Las acciones humanas por 
ejemplo, pierden el valor que tienen por lo que son en sí, aisladamente, y son vistas e interpretadas por la función que desempeñan en la estructura total de la personalidad. El acto humano se define por la red de relaciones que lo liga al todo. El método hermenéutico llega a ser así, el método por excelencia para la comprensión del comportamiento humano. (Martínez, 1989: 85-111)

Desde este paradigma podemos constatar que la tendencia al orden en los sistemas abiertos supera el carácter simplista de la explicación causal lineal y unidireccional y nos pone ante el hecho de la emergencia de lo nuevo y de lo imprevisto como fuentes de nueva coherencia. La ontología sistémica y su consiguiente metodología interdisciplinaria propuesta por este paradigma cambia radicalmente la conceptualización de toda entidad.

Desde el paradigma emergente se propone un enfoque modular, estructural, dialéctico, interdisciplinario y ético, en donde todo incide e interactúa con todo, en donde cada elemento no sólo se define por lo que es o representa en sí mismo, sino, y especialmente, por su red de relaciones con todos los demás. (Martínez, 1997: 24)

A partir de la perspectiva del "paradigma emergente" se plantea la problemática actual de cómo se ha venido construyendo el conocimiento de la ciencia, las implicaciones de corte epistemológico e ideológico en la construcción de conocimiento social que proporcionen las bases no sólo en el marco metodológico sino principalmente en el carácter formativo del investigador

\section{Los sistemas de información y documentación}

Luego de desarrollar brevemente las características que describen el paradigma emergente es conveniente realizar un sucinto análisis histórico de las perspectivas epistemológicas que ha adoptado la Ciencia de la Información:

1. Perspectiva neopositivista: hasta los años ochenta, la Ciencia de la Información adoptó el neopositivismo como enfoque epistémico para abordar los problemas de información y documentación, siguiendo los modelos matemáticos existentes, e imprimiendo en sus soluciones un carácter determinista, no dinámico e interactivo en el plano social, que excluía cualquier clase de elementos emocionales, afectivos o físicos y empleaban una metodología de investigación cuya naturaleza era cuantitativa. (Fernández \& Moya-Anegón, 2002: 244).

Esta concepción epistemológica prevalece aún en gran parte del imaginario colectivo social, mostrando el desconocimiento el dinamismo científico que la esta y otras disciplinas han adoptado desde hace aproximadamente treinta ańos.

2. Perspectiva cognitiva: Para Popper, un error característico de la filosofía moderna es su enfoque subjetivo que "interpretaba el conocimiento como una relación entre la mente subjetiva y el objeto conocido” (1974: 141), considerando a las expresiones simbólicas o lingüísticas como simples expresiones de estados de conciencia, que para el filósofo austriaco constituyen claramente un conocimiento objetivo. La visión propuesta por Popper, impulsó la incorporación de elementos cognitivos y sociales, que sin renunciar a las exigencias cuantitativas de la teoría de la información permitieran ampliar sus perspectivas teóricas. El principal aporte de este enfoque es la inclusión de estudios del comportamiento humano relacionados con la información. Los 
presupuestos básicos del enfoque epistémico cognitivo, se erigen sobre la diferenciación entre el carácter subjetivo y objetivo de la información, que consisten en primer lugar, en concebir el conocimiento como una configuración de estados mentales subjetivos dentro del individuo; y en segundo lugar, el conocimiento objetivo, que puede ser sometido a discusión crítica.

3. Perspectiva sociológica: Con la perspectiva cognitiva, se dan los primeros pasos para identificar otros planteamientos epistemológicos, diferentes a los propuestos por las ciencias naturales. Así, como lo explicamos anteriormente, el paradigma emergente, surge a partir del encuentro, la convergencia y el empleo de diferentes formas de análisis y cruce de perspectivas.

El aplicar el pensamiento sistémico y complejo en fenómenos de estudio como la información, la necesidad de información de los usuarios, los documentos, las Instituciones Informativas y el conocimiento, demandan que no se tire por la borda los conocimientos alcanzados por el paradigma clásico moderno, sino que sean retomados a través de nuevas perspectivas.

El pensamiento sistémico asume dos puntos de vista en sus comprensiones y descripciones, sin perder de vista que simultáneamente es un método de aprehensión y comprensión, así como de indagación y explicación: a) La clásica, en la que se "conoce el todo con el análisis o separación de las partes, pero no se observa el todo como interrelación de, entre las partes, como emergencia” (Rozo, 2003: 52); b) el sistémico y complejo, cuyo fin radica en la compresión de sistemas abiertos y dinámicos, en donde los elementos que los configuran reflejan interrelaciones, interacciones y conexiones, del sistema como un todo con el entorno y los sistemas en el entorno. (Rozo, 2003: 52)

Lo anterior aplicado a la Bibliotecología y Archivística, puede verse de forma más explícita retomando el esquema propuesto por Rendón (2008: 72-74), en donde reconoce, que un Sistema de Información y Documentación (SID) está conformado por la interacción de cuatro elementos esenciales: a) la información, b) el documento, c) el usuario y d) la Institución informativa documental. Concibe la información como resultado de la configuración del pensamiento, las ideas, los conceptos, los significados y los sentidos; considera al documento como la objetivación de la información bibliográfica en algún medio físico o simbólico; comprende al usuario como ser humano que tiene una necesidad de información que puede satisfacerse; y finalmente entiende a la Institución informativa documental como un ente social que proporciona las condiciones para satisfacer las necesidades de información de los usuarios.

Rozo (2003: 54), afirma que "un sistema está compuesto por una determinada cantidad de elementos heterogéneos, cuyas interrelaciones y conexiones configuran la emergencia de una unidad global". Además, establece una serie de parámetros que caracterizan los sistemas y que discutiremos brevemente estableciendo su aplicación a la Ciencia de la información.

1. Cada uno de los elementos constitutivos del sistema poseen un carácter subsistente, que implica la posesión de propiedades inherentes que hagan posible establecer similitudes y diferencias con otros elementos del sistema, y con la unidad sistémica global. Para el caso que estamos analizando, los elementos que confluyen en el SID, como ya lo hemos puntualizado, son la información, el documento, los usuarios -vistos desde sus necesidades de información-, y las Instituciones Informativas Documentales; 
cada uno de estos tiene interrelaciones con todos los demás, basta con analizar cómo confluyen por ejemplo en un fenómeno como la democratización del conocimiento, en donde la información se percibe como el resultado de toda actividad humana, que constituye la cultura, y que existe en forma independiente de nuestra reconocimiento personal, tal información requiere ser plasmada en algún tipo de documento, cuyo acometimiento ha de ser sustentable no solamente para la generación que más inmediatamente se beneficia por ser contemporánea al documento, sino además por todas las generaciones futuras, que han de analizar la información que contiene y las características que le son propias. Pero dicho análisis corresponde a las Instituciones Informativas documentales, que se encargan de interrelacionar cuáles son los procesos que permiten conservar los documentos, para que cumplan con la finalidad para la cual existen, que ha de ser la de poder ser consultados, pero así mismo es una tarea primordial de tales entidades identificar las necesidades reales de información de los usuarios y priorizar los mecanismos que puedan hacerlas posibles.

2. El sistema como unidad global no resulta de la suma de las características singulares de los elementos, sino de sus interrelaciones y conexiones, que son conocidas como intra-sistémicas. Esto se observa, al evidenciar que para que el SID, cumpla con su objeto propio, requiere identificar no solamente las características de la información contenida en los documentos, sino además la pertinencia y relevancia de la misma vista desde las necesidades de los usuarios y desde la proyección del desarrollo humano de una comunidad o sociedad específica, pero tal análisis ha de ser realizado por especialistas que hacen parte de lo que identificamos como Institución informativa Documental. Por tanto es la interrelación entre cada uno de los cuatro componentes con los otros tres, lo que configura el SID, imprimiéndole dinamismo. De allí que Capra afirme que "Lo que denominamos parte, es meramente un patrón dentro de una inseparable red de relaciones (...) En la visión sistémica vemos que los objetivos en sí mismos son redes de relaciones inmersas en redes mayores." (1996: 57).

3. "Las propiedades de las partes no son propiedades intrínsecas, sino que sólo pueden ser comprendidas en el contexto de un conjunto mayor (...) solo se puede comprender desde la organización del conjunto, por lo tanto, el pensamiento sistémico no se concentra en los componentes básicos, sino en los principios esenciales de la organización. El pensamiento sistémico es contextual, en contrapartida al analítico" (Capra, 1996: 48). Es el SID como Sistema Social el que brinda orientaciones esenciales a los elementos que lo componen, que hacen posible identificar y optimizar las interrelaciones. Se analiza la información desde un horizonte pragmático puesto que su existencia se debe a la actividad de un sujeto que está en potencia de ser usada por otro u otros sujetos; el documento es realizado con el fin de objetivar la información, para que asimismo pueda ser desobjetivada por otros individuos, haciendo posible el flujo de la información; el usuario identifica de forma específica una necesidad de información, cuya satisfacción puede exigirse a todo el sistema, ya sea a través de las bibliotecas o los archivos, o accediendo directamente al documento -si le es posible-; y la Institución Informativa Documental ha de proporcionar las condiciones necesarias para llevar a buen término todos los procesos que implica el SID.

4. La dificultad de descubrir un orden que oriente las relaciones e intercambios entre los elementos del sistema y su entorno, generan un sinnúmero de posibilidades y probabilidades que requieren ser tenidas en cuenta para tomar decisiones que vayan en 
la misma línea de su objeto. Con mayor razón, si nos detenemos a observar el impacto que han tenido las tecnologías de la Información en la generación, representación, recuperación y distribución de la información, dando pie a la configuración de nuevos conceptos de almacenamiento, edición, organización, transmisión y acceso de la misma, y modificación de las formas de representar el conocimiento, ocasionando una descentralización acelerada de su producción, validación, difusión, organización, almacenamiento y consulta, que puede realizarse en diversos niveles, como el institucional, comunitario, grupal o individual. Todo esto muestra claramente la complejidad que se entreteje en las múltiples variables que confluyen, que expresan un claro oscuro, en donde por la misma interdisciplinariedad, habrán aspectos que nos resulten claros y otros totalmente ajenos.

\section{Referências bibliográficas}

Beynam, L. (1978). The emergent paradigm in science. Revision Journal,1, 2.

Bunge, M. (1996). La ciencia, su método y su filosofía. Bogotá: Panamericana.

Capra, F. (1985). El punto crucial. Barcelona, España: Integral.

Capra, F. (1996). La trama de la vida. Una nueva perspectiva de los sistemas vivos. Barcelona, España: Anagrama.

Fernández, J. C. \& Moya-Anegón, F. (2002). Perspectivas Epistemológicas humanas en la documentación. Revista Española de Documentación Científica, 25, 241-253.

Hahn, E. \& Schilp, A. (1988) The Phiosophy of W,V.Quine. Illinois, E.E.U.A.: La Salle.

Jara, M. I. (2004). Emergencia y Sorpresa. Causalidad o emergencia: Diálogo entre filósofos y científicos. Bogotá, Colombia: Universidad de la Sabana, 123-137.

Kuhn, T. (1992). Las estructuras de las revoluciones científicas. México, México: Fondo de Cultura Económica.

Mancipe, E. \& Lukomski, A. (2008). Ciencia de la Información: Herramientas teóricas para su comprensión como Ciencia Social. Códice, 4, 1 (En edición).

Martínez, M. (1989). El método hermenéutico-dialéctico en las ciencias de la conducta. Anthropos 18, 85-111.

Martínez, M. (1997). El paradigma emergente. Hacia una nueva teoría de la racionalidad científica.. México, México: Trillas.

Martínez, M. (oct. - dic., 2006). Pertinencia social en la investigación endógena. Espacio Abierto, 15, 4, 725-740.

Morales, E. (2006). Diversidad, pluralidad e información: Una riqueza multicultural. Documentación de las Ciencias de la Información, 29, 325-334.

Morin, E. (1984). Ciencia con consciencia. Barcelona, España: Anthropos, Editorial del hombre.

Morin, E. (1985). Introducción al pensamiento complejo. Barcelona, Espańa: Gedisa.

Morin, E. (1986). El conocimiento del Conocimiento. Madrid, España: Cátedra.

Pigem, J. (1991). Nueva conciencia. Barcelona, Espańa: Integral.

Popper, K. R. (1994). Conjeturas y refutaciones. El desarrollo del conocimiento científico. Barcelona, España: Paidos.

Popper, K. R. (1974). Conocimiento Objetivo, (C. Solís, Trad.). Madrid, España: Tecnos.

Popper, K. R. (1985).Teoría cuántica y el cisma en la física. Madrid, España: Tecnos. 
Prigogine, I. \& Stengers I. (1986). La nouvelle alliance: metamorphose de la science. Paris, Francia: Gallimard.

Prigogine, I. (1988). Tan solo una ilusión? Una exploración del caos al orden. Barcelona, España: Tusquets Editores.

Rendón, M.A. (1998). Bases teóricas y filosóficas de la Bibliotecología. México, México: Universidad Nacional Autónoma de México.

Rendón, M.A. (ene. - jun., 2000). La ciencia bibliotecológica y de la información ętradición o innovación en su paradigma científico? Investigación Bibliotecológica, 14, 28, 34-51.

Rendón, M. (ene. -abr., 2008). Ciencia bibliotecológica y de la información en el contexto de las ciencias sociales y humanas. Epistemología, metodología e interdisciplina. Investigación Bibliotecológica. 22, 44, 65-78

Rozo, J. (2003). Sistémica y Pensamiento complejo: I Paradigmas, Sistemas y complejidad. Bogotá, Colombia: Biogénesis Fondo Editorial.

Ruiz, J. (2007). Metodología de la investigación cualitativa. Deusto, España: Universidad de Bilbao.

Setién, E. \& Gorbea, S. (ene. - jun., 1994). De la Bibliotecología al Sistema de Conocimientos Científicos Bibliológico-Informativo. Investigación Bibliotecológica, 8, 16, 21-25.

Snow, C. (1977). Las dos culturas y un segundo enfoque. Madrid, España: Alianza. 